\title{
Asymmetric Correlations between English Verb Transitivity and Stress
}

\author{
Michelle A. FullWoOd \\ Massachusetts Institute of Technology*
}

\section{Introduction}

It is well-known that lexical categories affect phonological behaviour (Smith 2011). Perhaps the most famous example of this is the stress asymmetry between nouns and verbs in English (Chomsky and Halle 1968): English disyllabic nouns tend to be trochaic while the verbs are iambic. Compare, for example, cómpress the noun with compréss the verb. This statistical tendency is extremely strong; according to Kelly and Bock (1988)'s lexical counts, 94\% of such nouns are trochaic, versus $31 \%$ of the disyllabic verbs.

A possible account for this stress asymmetry proposed by Kelly and Bock (1988) and Kelly (1988) is that lexical stress is influenced by prosodic environment. Nouns are more likely to occur in environments that favour trochaic stress since they often follow unstressed determiners, for example. Verbs are more likely to occur in environments that favour iambic stress since they are often followed by unstressed suffixes, among other factors.

This approach makes no direct reference to the lexical categories of noun and verb and in fact predicts that finer-grained lexical categories with differing prosodic contexts may display different stress behaviour. In particular, since transitive verbs are often followed by the unstressed determiner of their direct object, we might expect that transitive verbs are more likely to be iambic than intransitive verbs. I show in this paper that this prediction is borne out in English through a statistical analysis of English disyllabic verbs in the CELEX corpus (Baayen et al. 1995).

I then explore an additional mechanism by which prosodic context may influence word stress: the avoidance of phrase-final stress, which is attested cross-linguistically (Gordon 2000). I show that the likelihood for a disyllabic stem to be trochaic correlates with the frequency with which it occurs phrase-finally. I then propose a grammatical analysis based on Optimal Paradigms (McCarthy 2005) that synchronically captures these effects, and explore its typological predictions.

\section{Statistical analysis}

The CELEX English database (Baayen et al. 1995) provides phonological, morphological, syntactic and frequency data for (British) English lemmas and wordforms. Included among the syntactic data is verb subcategorization information, including two binary yes/no features

${ }^{*}$ Thanks to Adam Albright, Jennifer Smith, Morgan Sonderegger, Edward Flemming, and Norvin Richards, as well as audiences at the MIT Phonology Circle and Berkeley Linguistics Society, for discussion of this material. All infelicities are my own. This material is based upon work supported by the National Science Foundation Graduate Research Fellowship Program under Grant No. 1122374. 
Transitive ("is this a verb which can (sometimes) take a direct object?") and Intransitive ("is this a verb which (sometimes) cannot take a direct object?").

Based on these binary features, I encoded a ternary transitivity feature with the values obligatorily transitive, ambitransitive and obligatorily intransitive. Obligatorily transitive verbs were those with Transitive coded as $\mathrm{Y}$ and Intransitive coded as $\mathrm{N}$, such as appoint. Ambitransitive verbs were those with Transitive $\mathrm{Y}$ and Intransitive $\mathrm{Y}$. These included verbs that can optionally drop their direct object, such as eat (John ate the rice / John ate), and verbs that undergo causative alternations such as walk (Mary walked / Mary walked the dog). Obligatorily intransitive verbs had Transitive $\mathrm{N}$ and Intransitive $\mathrm{Y}$, such as chuckle.

The following table shows a simple lexical count of the disyllabic verbs of English, crossing the ternary transitivity distinction with stress pattern.

\begin{tabular}{l|cc} 
& Trochaic & Iambic \\
\hline Obligatorily transitive & $506(39 \%)$ & $804(61 \%)$ \\
Ambitransitive & $357(55 \%)$ & $293(45 \%)$ \\
Obligatorily intransitive & $227(64 \%)$ & $130(36 \%)$
\end{tabular}

Table 1: Percentage of verbs that are trochaic, split by transitivity type

We observe that the percentage of verbs that are trochees steadily increases as we move from obligatorily transitive to obligatorily intransitive verbs, with ambitransitive verbs being intermediate. Fisher's exact tests show that the percentage of verbs that are trochaic or iambic significantly differs by transitivity (obligatorily transitive versus ambitransitive: $p=$ $8.686 e^{-12} \ll 0.01$, ambitransitive vs obligatorily intransitive: $p=0.009<0.01$ ).

It is possible that this simple statistical test masks other confounds. In particular, stress may be influenced by the morphological structure of the verb. The following table, which splits up the verbs according to CELEX's morphological status field shows that while morphological status does have an effect on stress patterns, there are no significant reversals of the transitivity trend within each morphological category.

\begin{tabular}{|c|c|c|c|c|c|}
\hline \multirow[b]{2}{*}{ Morphological status } & \multicolumn{5}{|c|}{ Percentage trochaic } \\
\hline & $\begin{array}{l}\text { Oblig. } \\
\text { trans. }\end{array}$ & & $\begin{array}{l}\text { Ambi- } \\
\text { trans. }\end{array}$ & & $\begin{array}{l}\text { Oblig. } \\
\text { intrans. }\end{array}$ \\
\hline Monomorphemic & $49 \%$ & $<\mathrm{s}$. & $74 \%$ & $<$ n.s. & $75 \%$ \\
\hline Zero-derived & $82 \%$ & $<$ n.s. & $89 \%$ & $>$ n.s. & $80 \%$ \\
\hline Root-including & $15 \%$ & $<\mathrm{s}$ & $28 \%$ & $<\mathrm{s}$ & $57 \%$ \\
\hline Complex & $13 \%$ & $<\mathrm{s}$ & $47 \%$ & $<$ n.s. & $51 \%$ \\
\hline Obscure origin & $12 \%$ & $<$ n.s. & $16 \%$ & $<$ n.s. & $24 \%$ \\
\hline All verbs & $39 \%$ & $<\mathrm{s}$ & $55 \%$ & $<\mathrm{s}$ & $64 \%$ \\
\hline
\end{tabular}

Table 2: Percentage of verbs that are trochaic split by transitivity type and morphological status. (s. and n.s. indicate that the difference in percentages between successive percentages within a row were significant or insignificant according to a Fisher's test, respectively. 
Another factor that certainly has a influence on stress is the syllabic shape of the verb. To incorporate this into the statistical analysis, I conducted a logistic regression to predict whether a verb would be a trochee or iamb, with transitivity, morphological category and syllable shape and first-level interactions of morphological category and transitivity, and shape of syllable 1 and syllable 2 , as main factors.

The table below shows the main factors that were revealed to be significant. Positive estimates reveal that the factor is positively correlated with trochaicity. Hence, the positive estimate for the obligatorily intransitive factor shows that obligatory intransitivity significantly correlates with higher trochaicity when compared with ambitransitive verbs, while the negative estimate for the obligatorily transitive factor shows that obligatory transitivity significantly correlates with lower trochaicity when compared with ambitransitivity, even when the possible confounds of morphological category and syllable shape are controlled for.

\begin{tabular}{|ll|cccl|}
\hline Factor & & Estimate & Std Error & \multicolumn{2}{c|}{ Significance } \\
\hline \multirow{2}{*}{ Transitivity } & Obligatorily intransitive & 1.1096 & 0.4759 & 0.02 & $*$ \\
& Obligatorily transitive & -2.0291 & 0.3667 & $3.14 \mathrm{e}-08$ & $* * *$ \\
\hline \multirow{3}{*}{ Morphological status } & Complex & 1.4087 & 0.4273 & 0.00098 & $* * *$ \\
& Obscure & 3.8825 & 2.873 & 0.00406 & $* *$ \\
& Undetermined & 3.0771 & 0.4468 & $5.72 \mathrm{e}-12$ & $* * *$ \\
\hline \multirow{4}{*}{ Syllable 1 Weight } & VC & 0.5076 & 0.1788 & 0.00452 & $* *$ \\
& VV & 1.0916 & 0.2142 & $3.47 \mathrm{e}-07$ & $* * *$ \\
& VVC & 2.8359 & 0.3855 & $1.90 \mathrm{e}-13$ & $* * *$ \\
& VVCC & 3.7360 & 1.2065 & 0.00196 & $* *$ \\
\hline
\end{tabular}

Table 3: Significant factors in the logistic regression. Baselines were ambitransitive for the transitivity factor, monomorphemic for the morphological status category, and the lightest syllable weight of $\mathrm{V}$ for the syllable weight factors.

A nested model comparison via ANOVA of the factors in Table 2 with and without the two transitivity factors further confirms that the inclusion of transitivity significantly improves the fit of the model $(p=5.33 e-14<0.01)$.

To summarise the finding of this section, we find a significant effect of transitivity on the stress pattern of English disyllabic verbs, with transitive verbs being more likely iambic and intransitive verbs more likely trochaic.

\section{Grammatical analysis}

Kelly and Bock (1988) and Kelly (1988) suggest a possible account for the noun/verb stress asymmetry that is rooted in the prosodic environments in which nouns and verbs find themselves. Nouns are more likely to occur in an environment that favours trochaic stress, while verbs tend to occur in an environment that favours iambic stress. For instance, nouns are more likely to be preceded by unstressed elements such as determiners, while verbs are likely to be followed by unstressed elements, such as the determiner of a direct object. Verbs are 
also more likely to be followed by unstressed suffixes such as -ing and -ed, when the verb ends with $/ \mathrm{t}, \mathrm{d} /$.

This postulated tendency was borne out in corpus studies performed by Kelly and Bock (1988) and Kelly (1988), which found that there was indeed an increased tendency for nouns to occur in environments whose rhythmic requirements favoured trochaic stress and vice versa with iambs.

In addition, they performed pseudoword production experiments and found that speakers were more likely to pronounce a word with trochaic stress in a trochaic environment, and iambic stress in an iambic environment, as with the pseudoword pernew below.

(1) a. The bíg pernéw escáped.

b. Sáve the pérnew qúickly.

In proposing this account, Kelly and Bock (1988) and Kelly (1988) clearly had a diachronic mechanism in mind, wherein trochaic words that occurred in an iambic-favouring environment drifted towards iambicity via mispronunciations and misperceptions, as reflected in this quote:

"Over time, a word that consistently occupied a particular rhythmic context might come to reflect the pressures imposed by that context in its citation stress pattern." (Kelly and Bock 1988)

However, the results of the pseudoword experiments show that English speakers synchronically apply the effects of prosodic influence in generalising to novel forms, motivating a synchronic grammar of the noun/verb and transitivity asymmetries. The grammar I propose in this section is implemented via a MaxEnt weighted constraint model (Goldwater and Johnson 2003; Wilson 2006), since these effects are gradient rather than categorical.

In evaluating the output of a MaxEnt grammar for a given input and a set of constraints $C_{i}$ with weights $w_{i}$, we calculate first the harmony of each output candidate as the weighted sum of its constraint violations.

$$
w(x)=\sum_{i} w_{i} C_{i}(x)
$$

The probability of the grammar yielding a particular candidate $x$ as the winner out of a set of candidates $Y$ is then:

$$
P(x)=\frac{e^{-w(x)}}{\sum_{y \in Y} e^{-w(y)}}
$$

The particular constraints we will use in the analysis are introduced in the following subsections. 


\section{$3.1 *$ CLASH, *LAPSE}

The specific OT constraints governing the rhythmic effects of lexical environment are the familiar ${ }^{*}$ CLASH and ${ }^{*}$ LAPSE, which apply both word-internally and at the phrasal level.

(4) a. ${ }^{*} \mathrm{CLASH}: *$ for any pair of adjacent syllables that are both stressed.

b. *LAPSE: * for any pair of adjacent syllables that are both unstressed.

We have seen how the action of ${ }^{*}$ CLASH and ${ }^{*}$ LAPSE leads to the noun/verb asymmetry according to Kelly and Bock (1988) and Kelly (1988). Their account is easily extended to account for the transitivity asymmetry, borrowing directly from one of their own arguments: verbs are likely to be followed by an unstressed determiner of a direct object, but only when they are transitive.

While promising, this account is likely to be incomplete, as this effect is likely to be relatively small. In addition, there is no particular pressure on intransitive verbs to be trochaic.

One place to look for additional effects of prosodic environment on stress are among edge effects, which are not discussed in Kelly and Bock (1988) and Kelly (1988), but which are well known to have a large influence on lexical stress.

\subsection{NONFinAlity and * CROWD}

In fixed stress languages, there is an asymmetry between left and right edges (Hyman 1977; Gordon 2002). There are very few peninitial stress languages but a considerable number with penultimate stress. This is usually attributed to the effect of an OT constraint NonFinALity: do not stress the final syllable of a word.

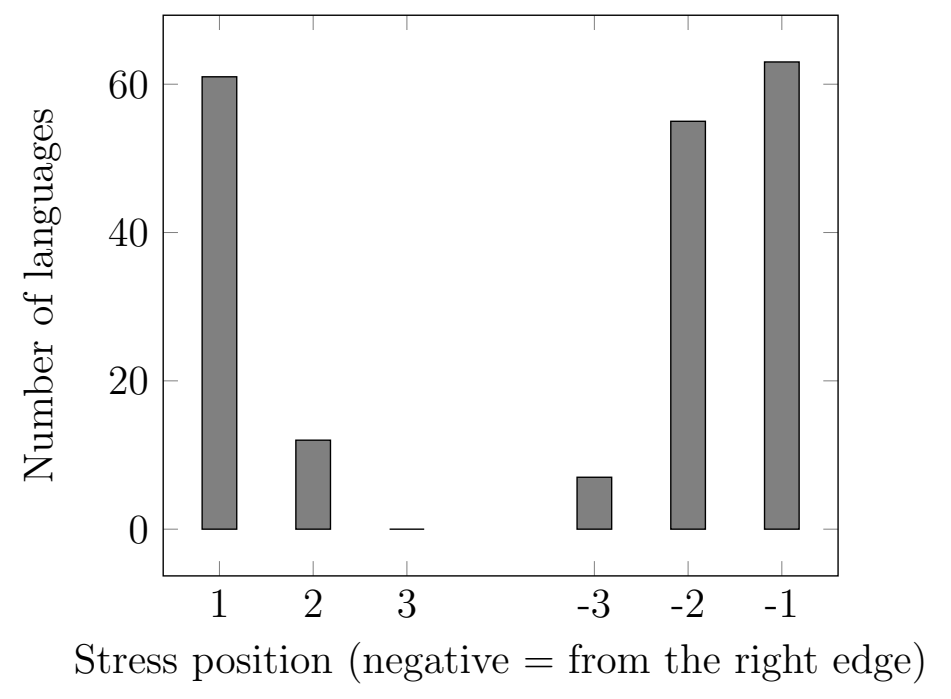

Figure 1: Graph showing number of languages with fixed stress in various positions at either edge of the word 
Gordon (2000) suggests a possible reason for the existence of NonFinaLiTy based on tonal crowding. Most, if not all, languages have right-edge boundary tones. If the final syllable of a phrase is stressed, both the phrasal boundary tone as well as the pitch accent due to stress would have to be accommodated on the same final syllable. Hence, there is a pressure to retract or remove stress from the phrase edge. We can define the constraint * Crowd to capture this particular pressure:

(5) *CROwD: * for any phrase-final syllable that is stressed.

How does phrase-level *CROwd lead to word-level NonFinality? Gordon (2000) hypothesises "that final stress avoidance at the word level is driven by phrase-final instantiations of words and that stress patterns of phrase-medial words are constrained to mimic their phrase-final counterparts." In languages where NonFinALITY appears to be undominated, as in the languages with consistent penultimate stress, this could be implemented via high-ranking output-output correspondence to the isolation form of the word.

In languages where output-output correspondence is ranked low while the constraint on tonal crowding is ranked high, on the other hand, we get a language where phrase-final syllables avoid stress, but phrase-medial word-final syllables do not. This is an attested pattern of behaviour in numerous languages such as Cayuga, Onondaga, varieties of Yupik, Chickasaw and Hill Mari (see Gordon (2000) and references therein).

I argue that English occupies an intermediate position between these two extremes, and that phrase-final frequency of individual words plays a role. Words that frequently occur phrase-finally are more likely to retract stress from their final syllable, while other words that rarely occur in phrase-final position are quite happy to accommodate a final stress.

\subsection{Testing for $*$ CROWD effects in English}

Since English word order is subject-verb-object, phrase-final items are more likely to be nouns than verbs. Similarly, intransitive verbs are far more likely to be phrase-final than transitive verbs. This can be verified through a count of 2.46 million words from the Penn Treebank's part-of-speech tagged Wall Street Journal and Brown corpora (Marcus et al. 1993). If we count the number of times a word stem appeared phrase-finally, that is, before a comma or sentence-finally, and not followed by a syllabic suffix such as -ed and -ing, and dividing it by the number of times the word appears in the corpus, we obtain the following average phrase-final frequency by part of speech:

\begin{tabular}{|c|c|}
\hline & Average phrase-final frequency \\
\hline Noun & $28.90 \%$ \\
Verb & $7.84 \%$ \\
\hline Intransitive verb & $14.16 \%$ \\
Ambitransitive verb & $8.43 \%$ \\
Transitive verb & $6.31 \%$ \\
\hline
\end{tabular}

Table 4: Average phrase-final frequency of different lexical categories in English within the Penn Treebank 
This count confirms that nouns and intransitive verbs are indeed more likely to appear phrase-finally than transitive verbs.

If English occupies this intermediate position between languages with across-the-board application of NonFinAlity and languages where only phrase-final syllables repel stress, we would expect both nouns and intransitive verbs to be more likely to retract stress, and hence for disyllabic nouns and intransitive verbs to be more frequently trochaic, which is precisely the pattern observed in $\S 2$.

A logistic regression shows that the phrase-final frequency of a disyllabic word is predictive of whether it will be a trochee or an iamb. When all 6224 nouns and verbs in the CELEX corpus are considered, the correlation is highly significant $(\mathrm{p}=2.34 \mathrm{e}-10<0.01)$.

When we isolate the 1772 disyllabic verbs in the corpus, however, the correlation between phrase-final frequency and trochaicity dips below significance $(\mathrm{p}=0.0531)$. A possible reason for this is that the phrase-final corpus frequency of low-frequency words is a very crude measure. Whether a certain word occurred in the corpus 100 times or a single time, if it never occurred word-finally, its phrase-final frequency would be $0 \%$. Yet it is clear that the former is a systematic effect while the latter could simply be an accident of the corpus, which might not be replicated in another corpus of similar size. If we exclude the lowest information words - those with only a single instance in the corpus - and repeat the logistic regression, the correlation becomes significant $(\mathrm{p}=0.01<0.05,1516$ verbs). The correlation retains significance when we add in the morphological status and syllable weight factors.

Since this approach is not tied to lexical category at all, we would expect to observe the effects of ${ }^{*}$ CROWD even within lexical categories. For example, a noun that tends to occur more as a direct object than a subject would have a higher phrase-final frequency and hence be more subject to ${ }^{*}$ CrOWD.

Repeating the logistic regression within only the class of disyllabic nouns that occur more than once in the Penn Treebank corpus, we find that the correlation between phrase-final frequency and trochaic stress is 0.055 - just above the threshold for significance. Within the class of ambitransitive verbs, the correlation stands at 0.0672 - again above the threshold for significance. There is no correlation between phrase-final frequency and stress pattern at all among the obligatorily intransitive and transitive verbs.

One reason for the failure of the noun correlation to reach significance could be that there are simply insufficient iambic nouns to make this determination, as $91.5 \%$ of the nouns remaining are trochaic. In the case of the three verb subclasses, there may simply be too few verbs (less than 1000 each) to make an accurate assessment of the correlation.

Despite the failure to find a significant effect of phrase-final frequency within lexical categories, the strong correlation within the entire set of disyllabic nouns and verbs, and within the class of all verbs, show that ${ }^{*}$ CROwD appears to be active in English, joining ${ }^{*}$ CLASH and * LAPSE as a markedness constraint governing prosodic environmental influence.

\subsection{Optimal Paradigms-based Faithfulness}

The effects of markedness are counteracted by an output-output faithfulness constraint. However, we have to determine what the base form with respect to which each candidate output will be evaluated. Unlike fixed stress languages, the base cannot be the citation form since we would then expect ${ }^{*}$ CROWD to apply equally to all words, regardless of lexical 
category. Indeed, we would expect the prosodic environment to have zero influence on the stress pattern of a word.

In fact, it is not clear that any particular form of a word in any particular context should be the base, if frequency is indeed a factor. Rather, what we need is a "voting" mechanism: if the various prosodic contexts in which a word finds itself tend to favour trochaicity, then all forms of the word are made trochaic.

To implement this, I borrow the technology of Optimal Paradigms (McCarthy 2005), with the following tenets.

(6) a. Candidates consist of the sets of occurrences of the word in its various lexical environments, with lexical stress on the word varying between candidates.

b. Markedness constraints evaluate all members of the set, and their violations are summed.

c. The word in each lexical environment is in a correspondence relation with the word in every other lexical environment, and thus syllables of the stem are in correspondence across lexical environments. When correspondent syllables do not share the same value of [ \pm stress], a violation of IDENT(stress)-OP is incurred. These violations are summed over all pairs of corresponding words.

The following sample tableaux illustrate the workings of the grammar.

(7) Sample MaxEnt tableau for the noun compress. Candidates, constraint counts, and their resultant harmony values and probabilities are purely illustrative.

\begin{tabular}{|c|c|c|c|c|c|c|}
\hline$/ \operatorname{compress}_{N} /$ & $\begin{array}{c}\text { IDENT-OP } \\
1.57\end{array}$ & $\begin{array}{c}{ }^{*} \text { CROWD } \\
0.71\end{array}$ & $\begin{array}{c}{ }^{*} \text { CLASH } \\
0.46\end{array}$ & $\begin{array}{c}* \text { LAPSE } \\
0.3\end{array}$ & $w(x)$ & $P(x)$ \\
\hline $\begin{array}{c}\text { the cómpress, } \\
\text { and cómpresses, } \\
\text { a cómpress for, ... }\end{array}$ & 0 & 0 & $\begin{array}{r}1 \\
1 \\
1 \\
1 \\
\end{array}$ & $\begin{array}{c}1 \\
1 \\
1 \\
1 \\
1\end{array}$ & 1.06 & 0.70 \\
\hline $\begin{array}{l}\text { the compréss, } \\
\text { and comprésses, } \\
\text { a compréss for, ... }\end{array}$ & 0 & 1 & $\begin{array}{c}1 \\
1 \\
1 \\
1\end{array}$ & $\begin{array}{l}1 \\
1 \\
1 \\
1\end{array}$ & 1.91 & 0.30 \\
\hline $\begin{array}{c}\text { the cómpress, } \\
\text { and comprésses, } \\
\text { a compréss for, ... }\end{array}$ & 4 & 0 & $\begin{array}{l}1 \\
1 \\
1\end{array}$ & $\begin{array}{l}1 \\
1 \\
1 \\
1 \\
1\end{array}$ & 6.88 & 0.00 \\
\hline
\end{tabular}

For each word with a similar profile of constraint violations, we expect the probability of it being trochaic to be $70 \%$. On average, the probability of a noun to be trochaic should be approximately 94\%, according to Kelly and Bock (1988)'s lexical counts.

On the other hand, the average transitive verb will have very different constraint violation profile, drastically lowering its likelihood to be trochaic, as in the following tableau: 
(8) Sample MaxEnt tableau for the transitive verb compress

\begin{tabular}{|c|c|c|c|c|c|c|}
\hline$/ \operatorname{compress}_{V} /$ & $\begin{array}{c}\text { IDENT-OP } \\
1.57\end{array}$ & $\begin{array}{c}{ }^{*} \text { CROWD } \\
0.71\end{array}$ & $\begin{array}{c}{ }^{*} \text { CLASH } \\
0.46\end{array}$ & $\begin{array}{c}* \text { LAPSE } \\
0.3\end{array}$ & $w(x)$ & $P(x)$ \\
\hline $\begin{array}{c}\text { Jóhn cómpresses the, } \\
\text { cómpressing, } \\
\text { I cómpress a, ... }\end{array}$ & 0 & 0 & $\begin{array}{ll}: & \\
\vdots & 1 \\
\vdots & \end{array}$ & $\begin{array}{ll} \\
\vdots \\
1\end{array}$ & 1.66 & 0.26 \\
\hline $\begin{array}{c}\text { Jóhn comprésses the, } \\
\text { compréssing, } \\
\text { I compréss a, ... }\end{array}$ & 0 & 0 & $\begin{array}{l}1 \\
\vdots \\
\vdots \\
1\end{array}$ & 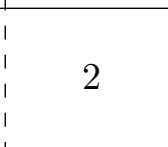 & 0.6 & 0.74 \\
\hline $\begin{array}{c}\text { Jóhn comprésses the, } \\
\text { compréssing, } \\
\text { I cómpress a, ... }\end{array}$ & 4 & 0 & 0 & 1 & 6.58 & 0.0 \\
\hline
\end{tabular}

Words with this constraint profile are iambic with a probability of $74 \%$.

The average intransitive verb would have a constraint profile intermediate between that of the average noun and the average transitive verb, resulting in an intermediate probability of being trochaic.

\subsection{Discussion}

One possible objection to this model is that unlike the original application of Optimal Paradigms to word paradigms, each candidate output set of lexical environments is extremely open-ended, making EvAL almost impossible. One possible solution is to sample a small number of exemplar lexical environments, making evaluation more manageable.

There is a further problem: even with IDENT(stress)-OP undominated, if EvAL is repeated every time a word is encountered in a new lexical environment, then we would still expect variation to occur. Say the verb compress is encountered once and the constraint evaluation in (8) carried out, and the iambic stress pattern is selected with $74 \%$ probability. If, the next time the verb compress is encountered, the trochaic stress pattern is selected instead, we would observe unwanted variation. To prevent this, we need to augment the grammar with a mechanism for storing the result of EvAL on each stem stress for future reuse.

This is a variant of the more general problem of how to account for exceptions to gradient phonotactic patterns. Several such mechanisms have been proposed in the literature (see, e.g., Zuraw (2000) for discussion). Another possible mechanism that might apply to this specific scenario is stochastic memoization, a probabilistic programming technique under which the results of previous computations are stored and reused with probability proportional to their frequency of past use, with a small amount of probability set aside for the generation of novel forms by the grammar (see O'Donnell (2011) for an application of stochastic memoization to regular and irregular morphology). Under this mechanism, we would expect frequently-encountered words to have a stable form and for novel forms to be generated according to the grammar. Low frequency lexical items would be more likely than frequent forms to display variation. 


\subsection{Predicted typology}

The language typology predicted by this model is one in which lexical category does not play a direct role in determining the stress of a word. Instead, the relative weighting of IDENT(stress)-OP, markedness constraints on rhythm and restricting stressed syllables at the end of an utterance from surfacing determine the probability with which a word receives a certain stress pattern.

This typology predicts two types of language when word-level stress constraints are not fully dominant. When markedness constraints such as * CROWD dominate IDENT(stress)-OP, the stress of individual word tokens adjusts to minimise markedness violations.

When IDENT(stress)-OP is undominated, we get English-type languages, where word types have a consistent stress, but the exact form is chosen to minimise overall markedness violations across lexical environments.

When * CROwD is high-ranked, barring interactions wth other phenomena, we predict that the English-type languages will split according to word order. In languages with SVO word order, nouns should be more trochaic than intransitive verbs, which are in turn more trochaic than transitive verbs. In languages with OVS, VOS and VSO word order, nouns should be more trochaic than verbs, but there will be no transitivity distinction, because utterances will almost always end with nouns. In languages with SOV and OSV order, verbs should be more trochaic overall than nouns, and again there should be no transitivity distinction since utterances will almost always end with verbs.

In languages with different distributions of unstressed elements than English, we also predict differences in stress patterns across lexical categories, although their effect is difficult to quantify overall. For example, if a language lacks determiners, then nouns have less pressure to be trochaic, and transitive verbs have less pressure to be iambic.

\section{Alternative accounts}

\subsection{Phonological grammars refer directly to lexical category}

An alternative to the account sketched above is that phonological grammars can simply refer to lexical category. This was the conclusion of Smith (2011), which surveys a wide range of category-specific effects across languages, and finds that while some effects may have their origin in factors such as the distinction between morphologically bound and free forms, these are unable to account for the entire gamut of category-specific effects. Such differences could be implemented via co-phonologies or via constraints indexed to lexical category. For example, the following simplified grammar could account for the majority patterns of English disyllabic stress. 


\begin{tabular}{|c|c|c|c|}
\hline Input: /compress (V)/ & NONFINALITY(noun) & Align-RIGHT & NONFINALITY \\
\hline cómpress & & $* !$ & \\
\hline b. compréss & & & $*$ \\
\hline Input: /compress (N)/ & NONFINALITY(noun) & ALIGN-RIGHT & NONFINALITY \\
\hline compréss & $* !$ & & * \\
\hline d. cómpress & & $*$ & \\
\hline
\end{tabular}

Extending this account to the transitivity asymmetry is straightforward. Phonological grammars should be able to go beyond lexical category, and make reference to verb transitivity, either via an explicit feature [ \pm transitive] that is accessible by the phonology, or by reference to the presence of some syntactic element corresponding to transitivity.

OT tableau for languish, intransitive verb

\begin{tabular}{|l||c|c|c|}
\hline Input: /languish/ (intrans) & NonFinality(intrans) & Align-Right & NonFinality \\
\hline \hline a. langúish & $* !$ & & $*$ \\
\hline b. lánguish & & $*$ & \\
\hline
\end{tabular}

This would result in a powerful framework capable of capturing any phonological differences due to lexical category or subcategory. However, unlike the prosodic environment account, this approach has no predictive power. There is no limit to the types of phonological behaviour that can be affected by lexical category, despite the fact that most cases of category-specific phenomena involve prosodic and suprasegmental features (Smith 2011). In addition, the direction of the asymmetry cannot be predicted: there is no particular reason for nouns to be more trochaic than verbs, nor for intransitive verbs to be trochaic than their transitive counterparts. The opposite patterns could be captured just as easily within this framework.

The predicted stress typology in this model is thus much less constrained than the one predicted in §3.6. Any lexical category can potentially display different stress behaviour, and we expect there to be no correlation with word order. There should also not be significant differences in stress behaviour within lexical categories apart from the usual effects of gradience.

\subsection{Phonological privilege correlates with prototypicality}

Among the generalisations Smith observes in her survey of category-specific effects (Smith 2011 ) is that nouns tend to display more phonological privilege than verbs, in the sense that nouns tend to support more phonological contrasts $(\mathcal{F} \gg \mathcal{M})$, while verbs undergo more neutralisation to unmarked structure $(\mathcal{M} \gg \mathcal{F})$. Based on this cross-linguistic tendency, Smith (2011) suggests that there is a hierarchy of phonological privilege, Noun $>$ Adjective $>$ Verb. 
In recent work, Smith (2013) proposes that this hierarchy correlates with a hierarchy of prototypicality, extending from prototypical arguments (nouns) to prototypical predicates (verbs). The notion of prototypicality stems from a body of work on the iconicity of lexical categories (e.g. Croft (1990); Hopper and Thompson (1985)).

Furthermore, Smith (2013) suggests that these broad lexical classes can be split into subclasses based on their position on the scale of prototypicality: for instance, unergative intransitive verbs are more prototypically predicate-like, being more agentive, than unaccusative intransitives, and thus unergative verbs should display less marked phonological behaviour than unaccusative verbs. Smith $(2013,2014)$ argues that this is the basis for a difference in the distribution of unaccentedness among unaccusative and unergative verbs in Tokyo Japanese.

This account cannot be straightforwardly extended to the English noun/verb stress asymmetry as the choice of trochaic or iambic stress pattern cannot be framed as a difference in phonological privilege. Neither the trochaic nor iambic stress pattern is more universally marked than the other - they simply obey different markedness constraints. Faithfulness plays no role.

If we take the slightly different approach of assuming that it is not phonological privilege but fidelity to markedness constraints such as Align-Stress-RIGHT that varies along the prototypicality scale, the noun/verb stress asymmetry suggests that in English, more prototypical predicates (verbs) are more strongly subject to Align-Stress-Right than more prototypical arguments (nouns). We would then expect that verbs that are less prototypically predicative should align with nouns in obeying ALIGn-STRESS-Right to a lesser degree. Hopper and Thompson (1985) suggest that transitive verbs are indeed more prototypically predicative than intransitive verbs, meaning that we correctly expect intransitive verbs to behave more like nouns in being more trochaic than transitive verbs.

Granting the assumptions made above, this approach only partially explains the stress asymmetries of English. It does not predict which particular markedness constraint(s) prototypicality correlates with. Instead of ALIGn-Stress-Right, we could have chosen NonFinALITY, and obtained the reverse stress asymmetry. The typology predicted by this approach is thus much less restrictive than that discussed in $§ 3.6$. It is slightly more restrictive than the typology predicted in $\$ 4.1$, in that stress behaviour does not vary freely with lexical category but is expected to pattern in a cline from nouns, to adjectives, to intransitive verbs, to transitive verbs, with the particular cline in a language dependent on which markedness constraints prototypicality correlates with. No correlations of stress behaviour with word order are expected, and no variation within lexical categories is expected, save where the lexical category can be split into further subcategories at different positions along the prototypicality scale.

\section{Conclusion}

In this paper, I have shown that going beyond the noun/verb stress asymmetry in English, there is a stress asymmetry between intransitive and transitive verbs: the former tend to be trochaic and the latter iambic. I showed how Kelly and Bock (1988) and Kelly (1988)'s theory that lexical stress is influenced by prosodic environment, originally proposed to extend to 
the noun/verb stress asymmetry, can be straightforwardly extended to the stress-transitivity asymmetry. I further augmented their proposal by illustrating that Gordon (2000)'s theory of NonFinality, when implemented within an Optimal Paradigms (McCarthy 2005) style "voting mechanism" for lexical stress, predicts that words that are more frequently phrasefinal are more likely to retract stress. A corpus study showed that there is indeed a positive correlation between phrase-final frequency and intransitivity, and between phrase-final frequency and trochaicity.

This correlation remains just a correlation, and not direct evidence for causation. Further work in the form of psycholinguistic experiments to test whether English speakers are aware of the stress-transitivity correlation and use avoidance of phrase-final stress in determining the stress of a nonce word, could help to elucidate the link between prosodic environment and transitivity as well as its synchronic status. Further evidence would come from a crosslinguistic investigation of other languages in which lexical stress and lexical category interact, to see if they fit into the typology predicted in $§ 3.6$.

\section{References}

Baayen, Harald R., Richard Piepenbrock, and Leon Gulikers. 1995. The CELEX lexical database. release 2 (CD-ROM). Philadelphia, Pennsylvania: Linguistic Data Consortium, University of Pennsylvania.

Chomsky, Noam, and Morris Halle. 1968. The Sound Pattern of English. New York, NY: Harper \& Row.

Croft, William. 1990. A conceptual framework for grammatical categories. Journal of Semantics 7:245-279.

Goldwater, Sharon, and Mark Johnson. 2003. Learning OT constraint rankings using a maximum entropy model. In Proceedings of the Workshop on Variation within Optimality Theory. Stockholm, Sweden: Stockholm University.

Gordon, Matthew. 2000. The tonal basis of final weight criteria. In Chicago Linguistics Society 36, 141-56.

Gordon, Matthew. 2002. A factorial typology of quantity insensitive stress. Natural Language and Linguistic Theory 20:491-552.

Hopper, Paul J., and Sandra A. Thompson. 1985. The iconicity of the universal categories 'noun' and 'verb'. In Iconicity in syntax, ed. John Haiman, 151-183. Amsterdam: John Benjamins.

Hyman, Larry. 1977. On the nature of linguistic stress. In Studies in stress and accent, ed. Larry Hyman, Southern California Occasional Papers in Linguistics 4, 37-82.

Kelly, Michael H. 1988. Rhythmic alternation and lexical stress differences in English. Cognition 30:107-137. 
Kelly, Michael H., and J. Kathryn Bock. 1988. Stress in time. Journal of Experimental Psychology: human perception and performance 14:389-403.

Marcus, Mitchell P., Beatrice Santorini, and Mary Ann Marcinkiewicz. 1993. Building a Large Annotated Corpus of English: The Penn Treebank. Computational Linguistics 19:313-330.

McCarthy, John J. 2005. Optimal paradigms. Linguistics Department Faculty Publication Series. Paper 55.

O'Donnell, Timothy J. 2011. Productivity and reuse in language. Harvard University dissertation.

Smith, Jennifer L. 2011. Category-specific effects. In The Blackwell Companion to Phonology. Blackwell Publishing.

Smith, Jennifer L. 2013. Lexical category effects in phonology: Whence and why? Handout from MIT colloquium talk.

Smith, Jennifer L. 2014. Prototypical predicates have unmarked phonology. In Supplemental Proceedings of the 2013 Meeting on Phonology, ed. John Kingston, Claire Moore-Cantwell, Joe Pater, and Robert Staubs.

Wilson, Colin. 2006. Learning Phonology with Substantive Bias: An Experimental and Computational Study of Velar Palatalization. Cognitive Science 30:945-982.

Zuraw, Kie Ross. 2000. Patterned exceptions in phonology. UCLA dissertation. 